\title{
Biological Characterization of Plasmodium falciparum Mitochondrial Heat Shock Protein PfHsp70-3: Possible Involvement in Malaria Pathogenesis
}

\author{
David O. Nyakundi ${ }^{1^{*}}$ and Aileen Boshoff ${ }^{2}$ \\ ${ }^{*}$ Mwenge Catholic University, P. O. Box 1226, Moshi - Tanzania. \\ E-mail: onchongadavid@hotmail.com; david.nyakundi@mwecau.ac.tz \\ ${ }^{2}$ Biotechnology Innovation Centre, Rhodes University, Makhanda/ Grahamstown 6140, \\ South Africa. E-mail: A.Boshoff@ ru.ac.za \\ Received 21 Jun 2021, Revised 22 Sep 2021, Accepted 25 Sep 2021, Published Oct 2021 \\ DOI: https://dx.doi.org/10.4314/tjs.v47i4.1
}

\begin{abstract}
Malaria remains a global health burden accounting for many deaths and illnesses in sub-Saharan Africa notwithstanding many decades of research on the disease. P. falciparum, the causative agent of the most fatal form of malaria, expresses a repertoire of heat shock proteins (Hsp) that cushion the parasite against heat shocks as it shuttles between extreme temperatures in human and mosquito vector hosts. By so doing, such proteins promote parasite's cytoprotection, survival and pathogenesis. Heat shock proteins are named according to their molecular weights and there are six P. falciparum Hsp70 (PfHsp70) found in various cell compartments with mitochondrial putative PfHsp70-3. Using indirect immunofluorescence, this study established mitochondrial localization of PfHsp70-3 though some more confirmatory studies would be needed in the future. PfHsp70-3 was found to be heat inducible and expressed during all stages of the intra-erythrocytic cycle of parasite development. This could be an indication of PfHsp70-3's involvement in the infectivity process of $P$. falciparum by helping the parasite to resist heat shocks during malaria febrile episodes. Generally, the data obtained in this study will enhance the existing knowledge on the biology of $P$. falciparum mitochondrial heat shock protein functions and open possible avenues for targeting the specificity between PfHsp70-3 and its co-chaperones for drug development.
\end{abstract}

Keywords: Malaria, P. falciparum, Heat shock proteins, PfHsp70-3, pathogenesis.

\section{Introduction}

Notwithstanding the great strides made in malaria control over the past decades, it still remains the most devastating infectious disease and major cause of morbidity and mortality to mankind (Murray et al. 2012, Molina et al. 2014). Nearly 1.2 billion people are at risk of malaria with 219 million new infections and 0.6 million deaths reported annually (Mendis et al. 2001, WHO 2015). $90 \%$ of reported malaria cases are entrenched in sub-Saharan Africa, affecting mostly children, pregnant mothers and the elderly (Rowe et al. 2009, Akachi and Atun 2011, WHO 2012). The spread of drug resistant parasite strains, inefficient vector control programs and lack of vaccine are among the factors worsening the effects of malaria and leading to negative economic development (Foley and Tilley 1998, Alonso et al. 2011). Human malaria is caused by unicellular protozoan parasites of the genus Plasmodium and transmitted by bites of infected female Anopheles mosquito; the most efficient of the sixty malaria-transmitting parasite vectors. The vector belongs to the phylum apicomplexan which replicate within the parasitophorous vacuole (Arrow et al. 2004). There are five eukaryotic Plasmodium 
species that cause malaria. They include $P$. falciparum, $P$. vivax, $P$. ovale, $P$. malariae, and P. knowlesi (Cox et al. 2010). Lately, based on molecular techniques, two species of $P$. ovale ( $P$. ovale wallikeri, and $P$. ovale curtisi) have been reported to infect humans (Calderaro et al. 2013). More than $90 \%$ of the world's malaria mortality is caused by $P$. falciparum which causes the most lethal form of cerebral malaria (Snow 2015). Incidental occurrence of both lethal P. falciparum and efficient Anopheline vectors in sub-Saharan regions of Africa, justify malaria endemicity in these regions (Zofou et al. 2014).

Malaria parasite life cycle involves two hosts; humans; where asexual reproduction takes place and female Anopheles mosquitoes; where the parasite reproduces sexually (Prudêncio et al. 2006). The infection cycle begins when sporozoites from an infected mosquito vector are injected into the human blood stream (Vaughan al. 2012). The sporozoites invade hepatocytes and undergo asexual reproduction to give rise to merozoites contained in merosomes (Duffy et al. 2012). The merozoites invade erythrocytes and initiate the second asexual reproduction phase allowing the parasite to develop from ring, trophozoite and finally to schizont stage (Baer et al. 2007 , Tilley et al. 2011). Schizont bursts and release merozoites to invade new erythrocytes (Duffy et al. 2012). Bursting and invasions of new erythrocytes result in malaria clinical symptoms characterized by high fever in patients (Cox-Singh and Singh 2010). A small percentage of merozoites differentiate into female and male gametes which circulate in the blood until they are taken up by a female anopheline mosquito when taking a blood meal to initiate the sexual phase inside the mosquito gut (Alano 2007, Baker 2010). Gametes mature and fertilization occurs to form a motile zygote (ookinete) within the lumen of the mosquito gut (Matuschewski 2006). The ookinete penetrates the gut and develops into oocysts which mature into sporozoites. Sporozoites migrate to the salivary glands of the mosquito ready to be injected into a new host when the mosquito feeds on blood. Malaria parasites experience heat shock episodes when shuttling between the cold blooded insect vector $\left(25^{\circ} \mathrm{C}\right)$ and warm blooded human host $\left(37{ }^{\circ} \mathrm{C}\right)$ (Sherman 1998, Bayoh and Lindsay 2003). A group of parasitic proteins commonly referred to as molecular chaperones also called heat shock proteins (Hsps) are believed to guarantee parasite survival by cushioning it from such temperature episodes. Molecular chaperones are a ubiquitous and evolutionarily conserved class of proteins that are essential for maintaining protein homeostasis (Powers et al. 2009). They function in a coordinated manner to prevent protein aggregation, refold and unfold proteins or direct them for degradation (Trougakos 2013). In particular, molecular chaperones assist newly synthesized proteins to reach their native conformational structure while protecting them from various types of stress such as extreme temperature (Bukau et al. 2006, Hartl and Hayer-Hartl 2009). The heat shock proteins are classified primarily on the basis of their molecular masses $(\mathrm{kDa})$ and sequence homology. The proteins are commonly abbreviated as "Hsp" when written, with the molecular weight indicated at the end, for example, Hsp70 (Heat shock protein with 70 kilo Daltons). However, due to the increased number of Hsps and discrepancies in their nomenclature, human Hsps have been renamed (Kampinga et al. 2009), with the names of the human proteins shown in parentheses. The major Hsps include Hsp110 (HSPH), Hsp90 (HSPC), Hsp70 (HSPA), Hsp60 (HSPD), Hsp40/J proteins (DNAJ) and small Hsp family (HSPB).

Clinical hallmarks of malaria are characterized by episodes of rise in body temperature, leading to increased Hsps expressions for cytoprotection (Pavithra et al. 2007, Pérez-Morales and Espinoza 2015). It therefore comes as no surprise that $P$. falciparum dedicates $2 \%$ of its total genome for encoding heat shock proteins (Acharya et al. 2007). The role of Hsps also becomes critical for the parasite during cellular stress induced by high protein turnover due to rapid 
multiplication rates in asexual blood stage development (Rathore et al. 2015). Heat shock proteins have also been shown to help the parasite to resist oxidative stress caused by antimalarial therapeutics by suppressing the host immune system (Akide-Ndunge et al. 2009). Furthermore, the asparagine (Asn) rich proteome of the parasite is vulnerable to aggregation and therefore requires $\mathrm{Hsps}$ to prevent probable aggregation (Aravind et al. 2003, Muralidharan et al. 2012).

A number of $P$. falciparum $\mathrm{Hsp} 70$ and Hsp40s have been proposed to play major roles in parasite development and survival and are therefore critical to the maintenance of cellular proteostasis through their roles in the folding, refolding, aggregation suppression, translocation and degradation of proteins (Shonhai et al. 2011, Njunge et al. 2013, Pesce and Blatch 2014). The P. falciparum genome encodes six Hsp70 (PfHsp70) homologues that localize in different cellular compartments where they carry out specialized and specific functions (Shonhai et al. 2011). PfHsp70-3 (PF3D7_113400) is predicted to reside in the mitochondria (Shonhai et al. 2007, Njunge et al. 2013). Mitochondrial Hsp70s serve as a motor system which interacts with pre-proteins to facilitate their entry into the matrix (Neupert and Brunner 2002). Mature proteins are then processed and properly folded upon entry into the mitochondrial matrix with the aid of $P$. falciparum chaperonin (Hsp60/Hsp10). Based on its signal peptide sequences, PfHsp70-3 has been proposed to reside in the mitochondria. However, previous reports had proposed it to occur in the Maurer's cleft where it possibly participates in exporting antigens to the erythrocyte surface (Vincensini et al. 2005, Shonhai et al. 2007). The vast majority of mitochondrial proteins are synthesized on cytosolic ribosomes and subsequently imported into the organelle as precursor proteins (Hutu et al. 2008). This requires mitochondrial resident chaperones and co-chaperones to assist in translocating imported protein across the membrane and refolding them into their conformational structure while in the matrix
(Neupert and Brunner 2002), and PfHsp70-3 plays a vital role in these processes. However, to date, there are no studies establishing the localization of the PfHsp70-3 to the parasite mitochondria. There are also no studies on its probable role in cushioning the parasite against heat shocks and its involvement in pathogenesis. Therefore, this study was carried out to establish the heat shock effects on PfHsp70-3, its expression profile during various intraerythrocytic developmental stages of P. falciparum and its possible mitochondrial localization.

\section{Materials and Methods \\ Sequence alignments of $\boldsymbol{P}$. falciparum Hsp70s and identification of antigenic determinant regions for antibody design \\ An antigenic region on PfHsp70-3 was} determined in order to develop antibodies for subsequent detection of the protein in parasite lysate. The amino acid sequence for PfHsp70-3 (PF3D7_1134000) was obtained from PlasmoDB v4.4 (Aurrecoechea et al. 2009) and analyzed using advanced antigen design algorithim GenScript's Optimum Antigen TM Design Program (GenScript, USA) to identify appropriate antigenic peptide sequences. The designing tool helps to establish and specify desired cross-reactivity, unexposed epitopes to be avoided, determination of the strength of antigenicity of chosen peptide, best conjugation and presentation options for desired assays and guaranteed immune response among others. Synthesis of the selected peptide and rabbit immunization was performed by GenScript (Hong Kong). Mitochondrial PfHsp70-3 was aligned with the known parasite Hsp70 proteins, i.e., PfHsp70-1 (cytosolic), PfHsp702 (endoplasmic reticulum), PfHsp70-X (exported) and PfHsp70-y and PfHsp70-z using Clustal omega. The alignment could ensure that the antigenic region selected was only unique for PfHsp70-3 and not any of the other five PfHsp70s, to avoid cross reactivity in subsequent experiments. 


\section{Determination of heat shock induction of PfHsp70-3}

To determine the heat inducibility of PfHsp70-3, P. falciparum parasites (clone 3D7) were cultured based on a previously described method (Trager and Jensen 1976), and synchronized at the ring stage. Once parasites had developed to trophozoites, the cultures were equally split into three flasks and heat shock was applied by incubating subcultures at $37{ }^{\circ} \mathrm{C}$ (control), $41{ }^{\circ} \mathrm{C}$ (heat shock) and $43{ }^{\circ} \mathrm{C}$ (heat shock) for 2 hours. Parasite pellets collected from the three subcultures were prepared by saponin lysis and analyzed through SDS-PAGE and western blotting. Anti-PfHsp70-3 antibody and HRPconjugated goat anti-rabbit secondary antibodies were used. Rabbit anti-actin antibody and uninfected erythrocytes were used as loading and negative controls, respectively.

\section{Analysis of intraerythrocytic time course expression of PfHsp70-3}

Infected $P$. falciparum parasites (clone 3D7) were cultured based on a previously described method (Trager and Jensen 1976), and synchronized at the ring stage with $5 \%$ sorbitol. The parasites were then split into six flasks and incubated at $37{ }^{\circ} \mathrm{C}$ for time course expression analysis. Infected erythrocytes were harvested by centrifugation at eight hour intervals over the 48 hour intra-erythrocytic parasite developmental life cycle. Parasite pellets obtained by saponin lysis were frozen at $-80^{\circ} \mathrm{C}$ for further analysis. The expression of PfHsp70-3 was analyzed by SDS-PAGE and immunodetection was carried out on the western blots using Clarity ${ }^{\mathrm{TM}}$ Western ECL blotting kit (Bio-Rad, USA) as per the manufacturer's instructions, and captured with a Chemidoc chemiluminescence imaging system (Bio-Rad, USA). Anti-PfHsp70-3 antibody and HRP-conjugated goat anti-rabbit secondary antibodies were used. Rabbit antiactin antibody (1:4000 dilutions) and uninfected erythrocytes were used as loading and negative controls, respectively.

\section{Indirect immunofluorescence microscopy}

Indirect immunofluorescence microscopy was carried out to determine the localization of PfHsp70-3 and was carried out as previously described by Tonkin et al. (2004). Infected erythrocytes were cultured and harvested at the trophozoite stage by centrifugation (2000 $\mathrm{g}$ for 3 minutes). The pellet of infected erythrocytes was re-suspended in RPMI (without Albumix) medium at room temperature. The mixture was added to the wells of poly-lysine coated chamber slides (Ibidi, Germany) followed by incubation at $37^{\circ} \mathrm{C}$ for 30 minutes to allow the red blood cells to settle on to the bottom of the slides. The unattached erythrocytes in the wells were gently washed off with RPMI medium and the formation of an attached erythrocyte layer on the bottom of the wells was confirmed by microscopy. The attached erythrocytes were rinsed twice in PBS containing $0.05 \%$ saponin to lyse the cells. Immediately $3 \%$ paraformaldehyde and $0.2 \%$ glutaraldehyde in PBS was added and incubated for 10 minutes. After incubation, the cells were washed with PBS followed by the addition of $0.2 \%$ Triton X-100 contained in PBS and incubated for 2 minutes. Glycine $(0.15 \mathrm{M})$ was added to block free reactive aldehyde groups that could react with antibodies followed by rinsing with PBS after incubation for 10 minutes. Blocking buffer (1\% BSA in PBS) was added to the fixed cells in the wells and incubated for 20 minutes. Anti-PfHsp70-3 (primary antibody), diluted 1:200 in blocking buffer, was added and allowed to bind for a minimum of 40 minutes. The cells were washed three times using washing buffer (PBS, 0.1\% BSA and $0.1 \%$ Tween 20 ) for $10 \mathrm{~min}$ each to remove excess primary antibody. FITC-conjugated goat anti-rabbit (secondary antibodies; SigmaAldrich) was added at 1:200 dilutions in blocking buffer and allowed to bind for 40 minutes in the dark. The wells were washed thrice by the wash buffer followed by the addition of 4,6-Diamidine-2-phenylindole dihydrochloride (DAPI) and incubated for 1 minute to bind to the DNA. The wells were rinsed with water, mounted under a coverslip in 
FluorPreserve (Calbiochem) and viewed with an Olympus BX60 epifluorescence microscope using $100 \times$ oil-immersion objectives.

\section{Results and Discussions}

The $P$. falciparum Hsp70s was aligned with other PfHsp70 homologues and an antigenic region for antibody design was determined. The sequence alignments of $P$. falciparum Hsp70s are shown in Figure 1.

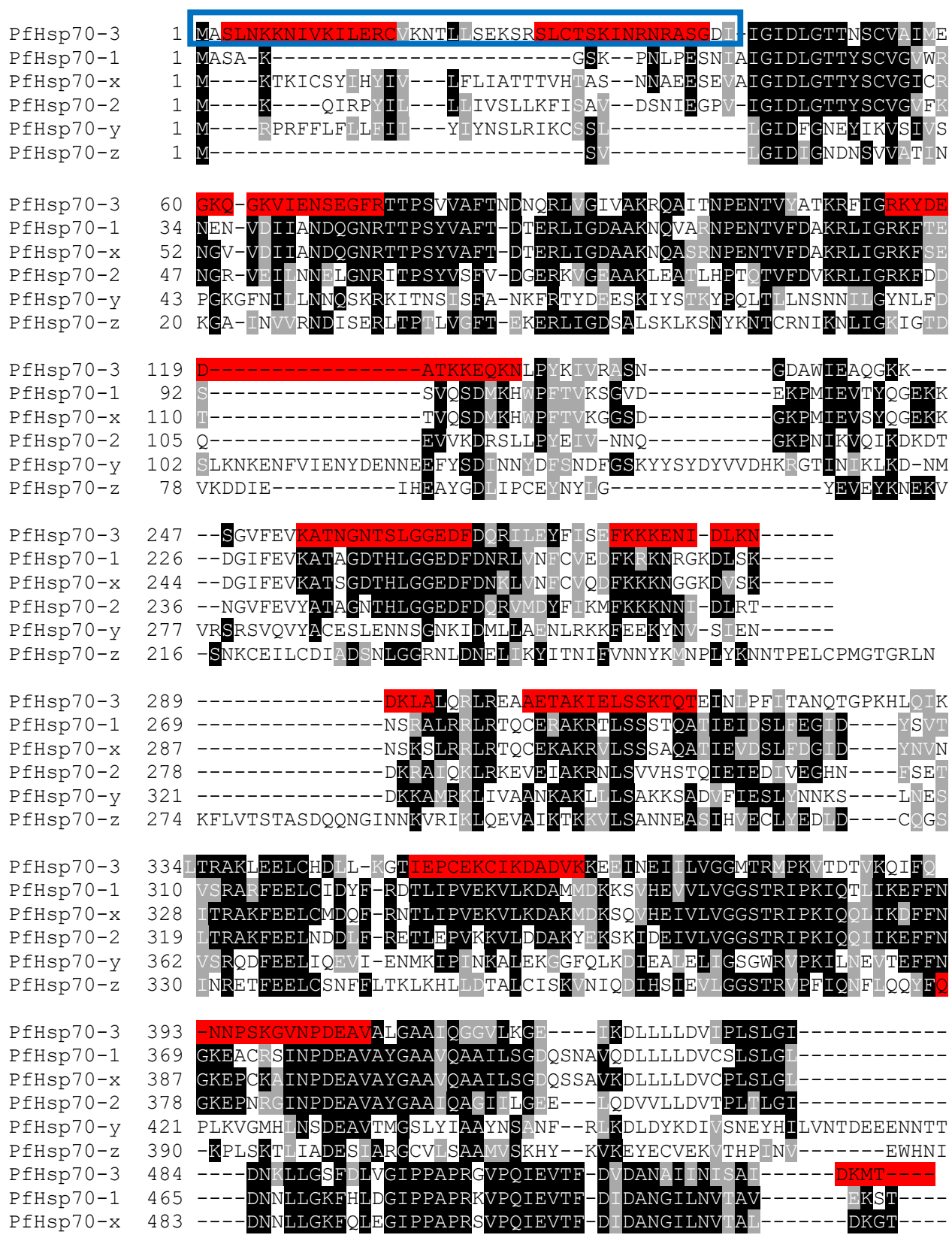




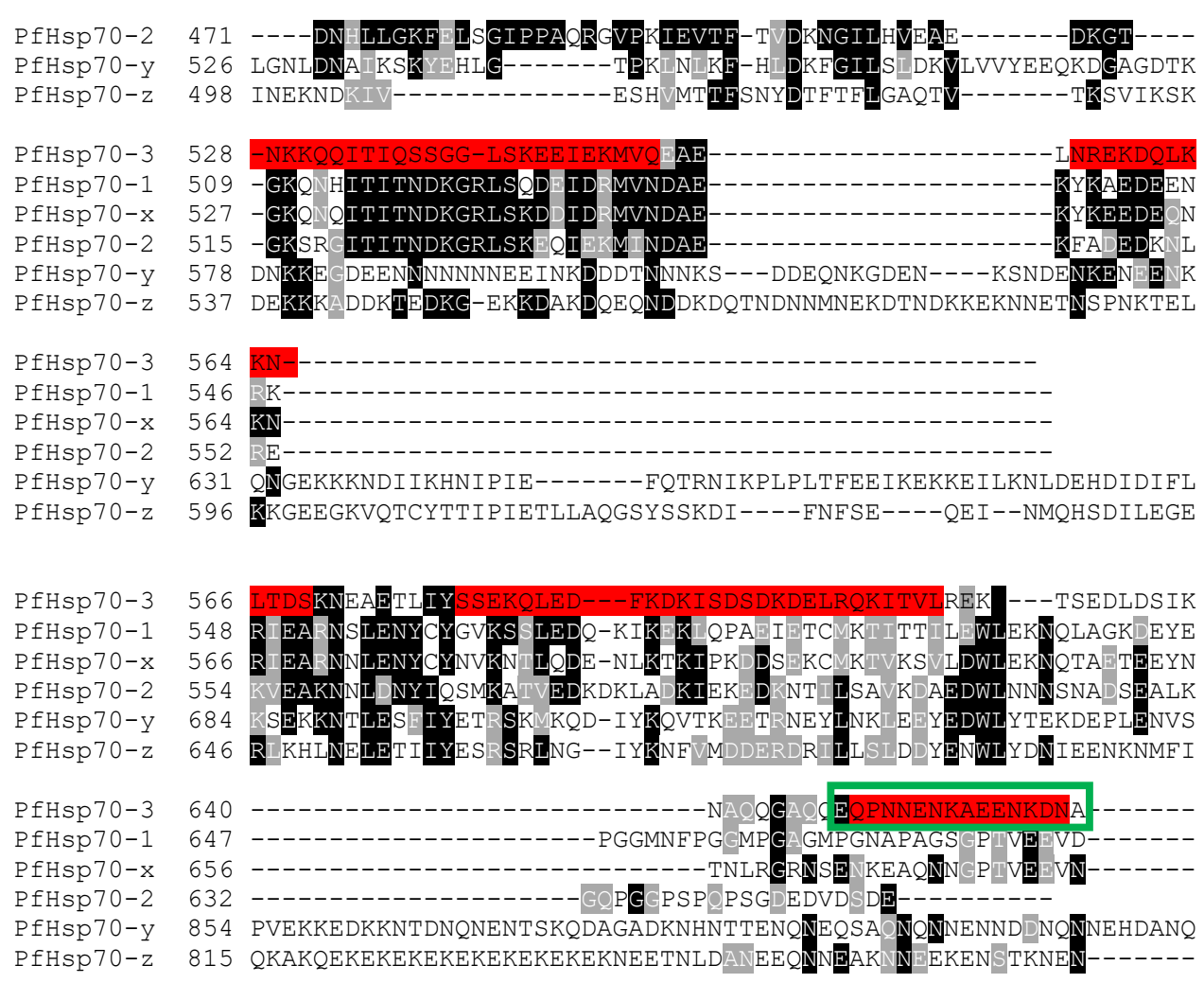

Figure 1: Sequence alignments of $P$. falciparum Hsp70s showing antigenic determinant regions in PfHsp70-3:PfHsp70-3 aligned with PfHsp70-1, PfHsp70-2, PfHsp70-x, and PfHsp70-y and PfHsp70-Z using Clustal omega. Red highlight: antigenic determinant regions, blue box: mitochondrial putative signal peptide sequence, green box: peptide used to raise specific antiserum against PfHsp70-3 in rabbit. The black shade represented $100 \%$ residue identity with dark gray representing similarity.

To select a suitable peptide antigen for antibody development, a number of aspects were duly considered that included antigenicity, hydrophilicity, hydrophobicity, surface probability, predicted transmembrane domains, homology, flexible region, helix region, sheet region, signal peptide and modification (Hofmann and Hadge 1987, Hopp and Woods 1981, Jameson and Wolf 1988). Most importantly, it was ensured that the peptide sequence selected was not similar to any of the other five Hsp70 homologues to avoid cross reactivity and non-specificity. The chosen peptide sequence was used as a query in the blast search and the sequence alignment confirmed that the region was unique to PfHsp70-3 (Figure 1 in green box).

Peptides that were located within the ATPase domain of the protein were avoided due to high conservation of the domain residues among the parasite Hsp70 proteins as compared to the highly variable C-terminal domain (Figure 1). Based on these factors, the peptide CQPNNENKAEENKDN was selected for antibody development in Oryctolagus cuniculus after performing local alignments against the $P$. falciparum proteome. The selected peptide was used to produce antibodies against PfHsp70-3. The specificity of the antibodies developed from selected peptide sequence was determined by the use of 
parasite lysate prepared from the trophozoite stage parasites (Figure 2, lane 1). A protein band of approximately $70 \mathrm{kDa}$ was detected at almost the same size of purified His-tagged PfHsp70-3 which was used as a positive control (Figure 2, lane 3). There was no band detected in the uninfected erythrocytes that was used as a negative control (Figure 2, lane 2).

\section{The expression of PfHsp70-3 was induced by heat shock treatment}

Protein detection confirmed that PfHsp70-3 was being expressed in the intra-erythrocytic stages of parasite development and this facilitated an analysis of its heat inducibility. A number of $P$. falciparum Hsps have been demonstrated to be up-regulated during heat shock. PfHsp70-3 was analyzed to determine whether it would be up-regulated in response to heat shock as an indication of its role in cytoprotection during infectivity. The western blot analysis indicated an upregulation of PfHsp70-3 corresponding with an increase in temperature (Figure 3). There were more proteins expressed when the parasite was subjected to temperature higher than body temperature $\left(37^{\circ} \mathrm{C}\right)$. The parasite was exposed to higher temperature assumed to be similar to those occurring during malaria febrile $\left(41^{\circ} \mathrm{C}\right.$, and $\left.43{ }^{\circ} \mathrm{C}\right)$. This observation implies that PfHsp70-3 could participate in protecting the parasite against heat stress, especially, during malaria fevers thus guaranteeing parasite survival during this period.

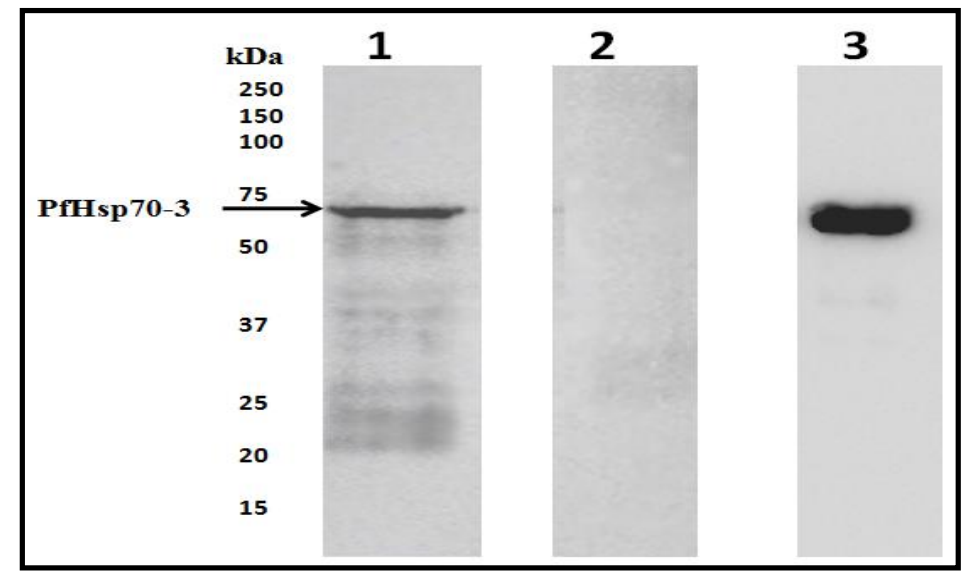

Figure 2: Specificity of anti-PfHsp70-3 peptide directed antibody and detection of PfHsp70-3 in parasite lysate: Western blot analysis of PfHsp70-3 using anti-PfHsp70-3 peptide directed antibody. Lane 1, detection of PfHsp70-3 in trophozoite parasite lysate; lane 2, uninfected erythrocytes, lane 3, purified His-tagged PfHsp70-3. 


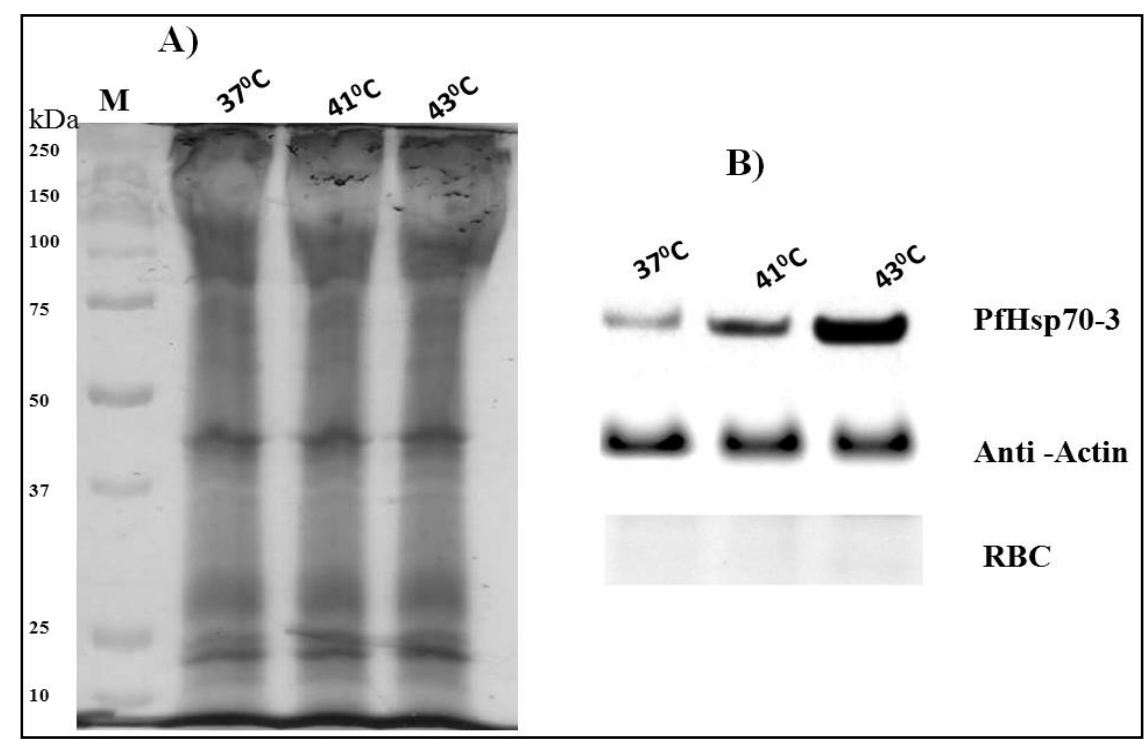

Figure 3: PfHsp70-3 is up-regulated following heat-shock: (A) SDS-PAGE (10\%) protein profiles of parasites incubated at $37{ }^{\circ} \mathrm{C}, 41{ }^{\circ} \mathrm{C}$, and $43{ }^{\circ} \mathrm{C}$ for 2 hours. (B) Western analysis: detection of PfHsp70-3 and actin from saponin-lysed parasite lysates obtained from 3D7 P. falciparuminfected erythrocytes using anti-PfHsp70-3 and anti-actin antibodies, respectively. Equal amount of infected erythrocytes were loaded per lane and three independent experiments were carried out and similar results were obtained.

\section{Maximum expression of PfHsp70-3 occurs} during the trophozoite stage

Having established upregulation of PfHsp70-3 against increased temperature, an expression profile of PfHsp70-3 was carried out over 48 hours of intra-erythrocytic development of $P$. falciparum. This was to determine when the maximum expression of PfHsp70-3 protein occurs during developmental stages. The parasite lysates were analyzed at eight hour intervals following sorbitol synchronization at ring stage. The SDS-PAGE and western analyses of equal numbers of infected erythrocytes showed different protein expression profiles at each developmental stage of the parasite (Figure 3). Actin was used as a loading control to confirm equally equivalent loading in each lane. The results showed that the protein was being expressed at all stages of $P$. falciparum development. There was least expression of the protein at the ring stage and the expression increased gradually with maximum expression occurring at early and late trophozoite stages (Figure 3). The observed expression profile of PfHsp70-3 appeared to correlate with mRNA expression profiles of the protein as earlier described and documented in PlasmoDB (Aurrecoechea et al. 2009). 


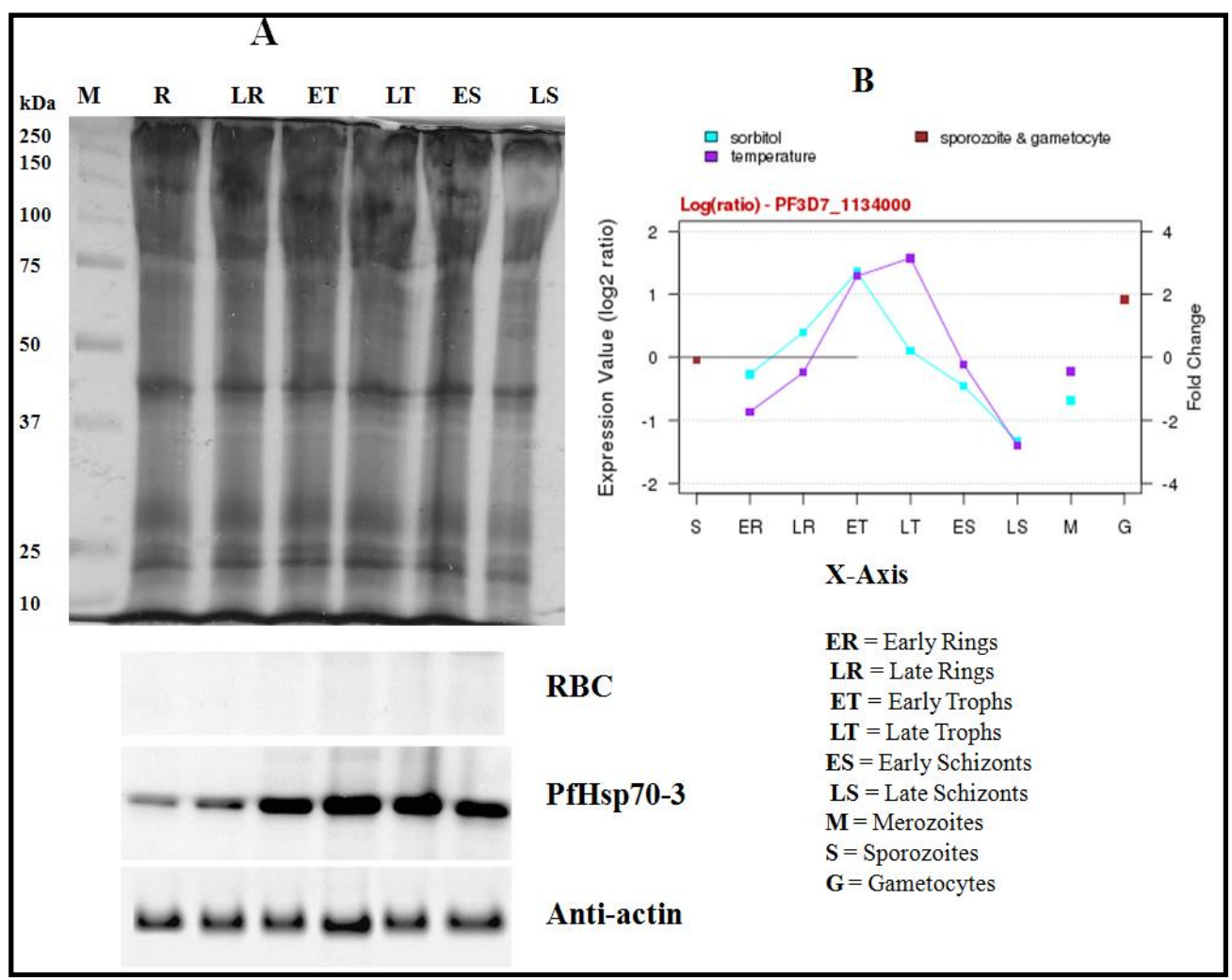

Figure 4: Maximum expression of PfHsp70-3 occurs at trophozoite stage in intraerythrocytic cycle of parasite development: A) SDS-PAGE (10\%) indicating PfHsp70-3 protein expression profile at different time points with corresponding western blot analysis (WB) probed for PfHsp70-3 and actin proteins using respective antibodies. Non-infected erythrocyte extract (RBC) was loaded as a negative control. (B) Messenger RNA expression profile of PfHsp70-3 during different phases of development of the parasite as adapted from PlasmoDB version 4.4 (Aurrecoechea et al. 2009). Equal numbers of infected erythrocytes were loaded per lane and three independent experiments were carried out and similar results were obtained.

PfHsp70-3 localizes to the $P$. falciparum mitochondrion

Localization of endogenous PfHsp70-3 was carried out using the antibodies through indirect immunofluorescence microscopy of trophozoite and early schizont-infected erythrocytes immobilized on poly-lysine coated slides. The erythrocytes were briefly lysed with saponin to improve antibody access. Polyclonal antibodies that were raised in rabbit against a C-terminal peptide "CQPNNENKAEENKDN" of PfHsp70-3 protein coding sequence were used as primary antibodies to investigate the localization of PfHsp70-3. FITC-conjugated goat anti-rabbit secondary antibodies were used. After immobilization and saponin lysis, paraformaldehyde/ glutaraldehyde fixation was used to fix the parasites before antibody binding.

Using the secondary green fluorescence signal, an elongated structure was observed at the periphery of the parasite believed to be the parasite mitochondrion (Figure 4). These preliminary findings indicated that PfHsp70-3 could indeed localize to the parasite mitochondrial organelle. Due to the delicate 
nature of the method of parasite fixation and lysis, most of the images observed showed broken and discontinuous fluorescence signal (data not shown). A fluorescence signal was absent in the negative controls where a primary or secondary antibody only was used (data not shown).

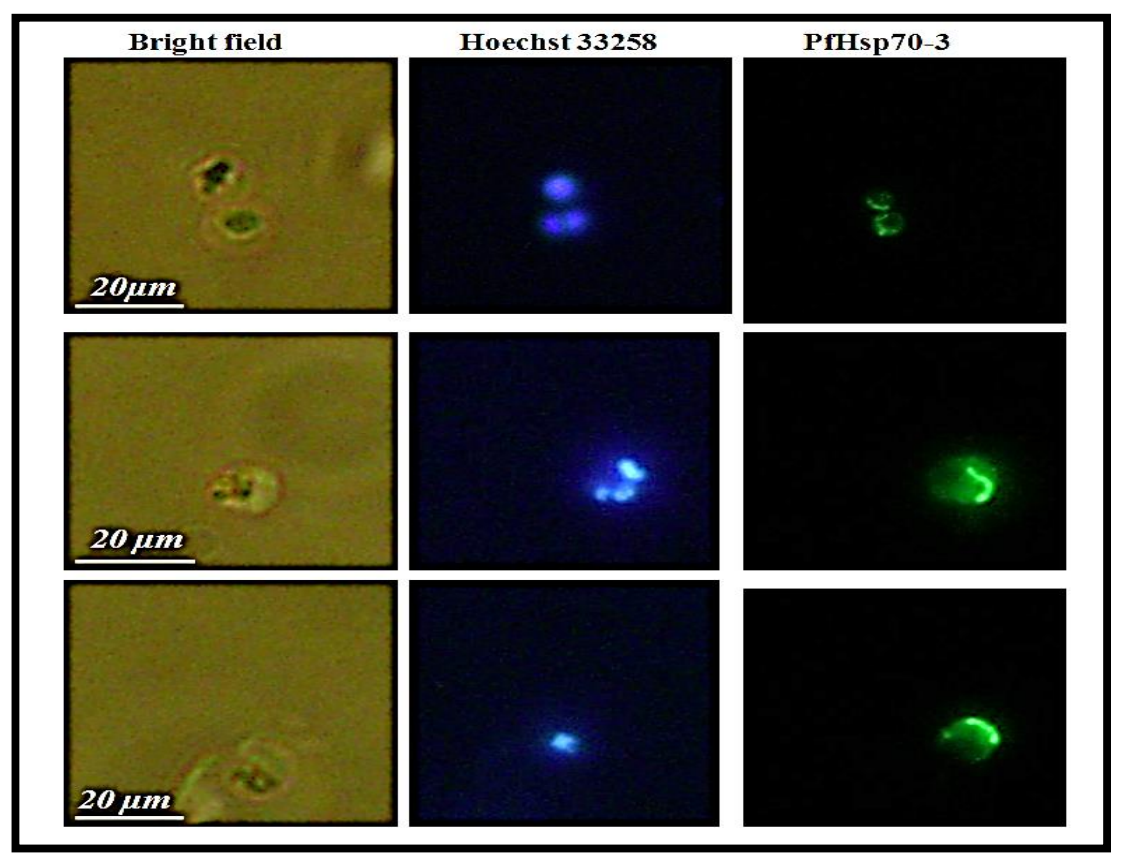

Figure 5: PfHsp70-3 localizes to the mitochondrion in P. falciparum: Sample of images obtained from localization study with rabbit anti-PfHsp70-3 peptide antibody on paraformaldehyde/glutaraldehyde-fixed $P$. falciparum trophozoites. Rabbit anti-peptide primary and anti-rabbit FITC-conjugated secondary antibodies was used to detect PfHsp70-3 protein. The parasite nuclei were stained with Hoechst 33258 (in blue) and the bright field (BF) showed the bright field images of the fixed parasites. The experiment was repeated at least three times and representative images are shown here.

\section{Conclusion and Recommendations}

In silico analysis revealed that the PfHsp703 protein contains a mitochondrial signal peptide sequence, an indication of its mitochondrial localization. However, this had not been experimentally validated and this study examined the cellular localization of PfHsp70-3. Indirect immunofluorescence microscopy studies on the localization of PfHsp70-3 indicated that the protein could be localized to the parasite mitochondrion. A fluorescence signal was observed around an elongated structure at the exterior parts of the parasite nucleus that was consistent with mitochondrial morphology. However, since both the parasite mitochondrion and the apicoplast are found lying side by side at this stage of development (Siregar et al. 2015), differential staining using markers for the two organelles would be required in order to be conclusive on the exact localization of PfHsp70-3. Alternatively, a confirmation of localization can be done through isolation of parasite mitochondrial fraction and subsequent use of the antibodies against PfHsp70-3 to confirm localization. The use of PfCytochrome 
$\mathrm{C}$ as a mitochondrial marker and PfUROD as an apicoplast marker have been used to determine the localization of PfHsp60. The approach could be used for confirmatory PfHsp70-3 mitochondrial localization.

Transcription analysis of PfHsp70-3 had revealed the highest PfHsp70-3 mRNA expression levels during the late stages of the $P$. falciparum life cycle, with maximum expression occurring during the trophozoite stages of development. This study investigated protein expression of PfHsp70-3 to determine whether it correlated with the reported mRNA expression pattern. The analysis of parasite lysates at different stages of parasite development using anti-PfHsp70-3 antibodies, revealed PfHsp70-3 proteomic expressions that correlated with the transcriptomic profile. PfHsp70-3 protein expression progressively increased from the ring stage of the asexual development and attained maximum levels during the late trophozoite stage. $P$. falciparum parasites encounter stressful environmental changes during their establishment in the human host. Indeed, temperature variations characterize $P$. falciparum life cycle raging from the low temperature in the cold blooded vector, anopheles mosquito at $37{ }^{\circ} \mathrm{C}$ in healthy human hosts and $41{ }^{\circ} \mathrm{C}$ in patients suffering from malaria infection during febrile episodes. Heat shock treatment of $P$. falciparum parasites at $41{ }^{\circ} \mathrm{C}$ and $43{ }^{\circ} \mathrm{C}$ for two hours, visibly increased the expression of PfHsp70-3 compared to the expressions at $37^{\circ} \mathrm{C}$. Increased expression of PfHsp70-3 due to heat induction could imply the involvement of the protein in the heat-stress response mechanism of the parasite that is aimed at cushioning the parasite proteins against stressful conditions that could lead to aggregation and inactivity of such proteins. In conclusion, this study observed that PfHsp70-3 protein is expressed during all parasite asexual developmental stages with maximum expression occurring at trophozoite stages. The expression pattern of PfHsp70-3 at all infective stages could provide further evidence of the protein's involvement in malaria disease development. This could qualify PfHsp70-3 as a possible drug target candidate in the ongoing research on efficient malaria therapeutics.

\section{Acknowledgement}

The author would like to acknowledge the Department of Biochemistry and the Biotechnology Innovation Center at Rhodes University-South Africa, for making available the lab facilities upon which the experiments were conducted. The authors also acknowledge Prof. Heinrich Hoppe of Rhodes University for assistance in experimental set up and interpretation of the results.

\section{Competing Interest}

The authors of this manuscript declare that they have no competing interests.

\section{References}

Acharya P, Kumar R and Tatu U 2007 Chaperoning a cellular upheaval in malaria: Heat shock proteins in Plasmodium falciparum. Mol. Biochem. Parasitol. 153(2): 85-94.

Akachi Y and Atun R 2011 Effect of investment in Malaria control on child mortality in Sub-Saharan Africa in 20022008. PLoS One 6(6): e21309.

Akide-Ndunge OB, Tambini E, Giribaldi G, McMillan PJ, Müller S, Arese P and Turrini F 2009 Co-ordinated stage-dependent enhancement of Plasmodium falciparum antioxidant enzymes and heat shock protein expression in parasites growing in oxidatively stressed or G6PD deficient red blood cells. Malar. J. 8: 113.

Alano P 2007 Plasmodium falciparum gametocytes: Still many secrets of a hidden life. Mol. Microbiol. 66: 291-302.

Alonso PL, Brown G, Arevalo-Herrera M, Binka F, Chitnis C, Collins F, Doumbo OK, Greenwood B, Hall BF, Levine MM and Mendis K 2011 A research agenda to underpin malaria eradication. PLOS Medicine 8(1): e1000406.

Aravind L, Iyer LM, Wellems TE and Miller LH 2003 Plasmodium Biology: Genomic 
Gleanings. Cell 115: 771-785.

Arrow KJ, Panosian C and Gelband H 2004 Saving Lives, Buying Time: Economics of Malaria Drugs in an Age of Resistance, Institute of Medicine (US) Committee on the Economics of Antimalarial Drugs. ISBN-10: 0-309-09218-3.

Aurrecoechea C, Brestelli J, Brunk BP, Dommer J, Fischer S, Gajria B, Gao X, Gingle A, Grant G, Harb OS, Heiges M, Innamorato $\mathrm{F}$, Iodice $\mathrm{J}$, Kissinger $\mathrm{JC}$, Kraemer E, Li W, Miller JA, Nayak V, Pennington C, Pinney DF, Roos DS, Ross C, Stoeckert CJ, Treatman $\mathrm{C}$ and Wang $\mathrm{H}$ 2009 PlasmoDB: A functional genomic database for malaria parasites. Nucleic Acids Res. 37(Suppl. 1): D539-D543.

Baer K, Klotz C, Kappe SHI, Schnieder T and Frevert U 2007 Release of hepatic Plasmodium yoelii merozoites into the pulmonary microvasculature. PLoS Pathog. 3: e171.

Baker DA 2010 Malaria gametocytogenesis. Molecular and Biochemical Parasitology 172: 57-65.

Bayoh MN and Lindsay SW 2003 Effect of temperature on the development of the aquatic stages of Anopheles gambiae sensu stricto (Diptera: Culicidae). Bull. Entomol Res. 93(5): 375-381.

Bukau B, Weissman J and Horwich A 2006 Molecular chaperones and protein quality control. Cell 125: 443-451.

Calderaro A, Piccolo G, Gorrini C, Rossi S, Montecchini S, Dell'Anna ML, De Conto F, Medici MC, Chezzi C and Arcangeletti MC 2013 Accurate identification of the six human Plasmodium spp. causing imported malaria, including Plasmodium ovale wallikeri and Plasmodium knowlesi. Malar. J. 12(1): 321.

Cox FE 2010 History of the discovery of the malaria parasites and their vectors. Parasites \& vectors 3 (1): 5.

Cox-Singh J and Singh B 2010 Europe PMC Funders Group Knowlesi malaria: newly emergent and of public health importance? Trends Parasitol. 24(9): 406-410.
Duffy EP, Sahu T, Akue A, Milman N and Anderson C 2012 Pre-erythrocytic malaria vaccines: identifying the targets. Expert Rev. Vaccines 11(10): 1261-1280.

Foley M and Tilley L 1998 Quinoline antimalarials: mechanisms of action and resistance and prospects for new agents. Pharmacol. Ther. 79(1): 55-87.

Hartl FU and Hayer-Hartl M 2009 Converging concepts of protein folding in vitro and in vivo. Nature Structural and Molecular Biology 16(6): 574-581.

Hofmann HJ and Hädge D 1987 On the theoretical prediction of protein antigenic determinants from amino acid sequences. Biomed. Biochim. Acta 46(11): 855-866.

Hopp TP and Woods KR 1981 Prediction of protein antigenic determinants from amino acid sequences. Immunology 78(6): 38243828.

Hutu DP, Guiard B, Chacinska A, Becker D, Pfanner N, Rehling P, and van der Laan M 2008 Mitochondrial protein import motor: differential role of Tim44 in the recruitment of Pam17 and J-complex to the presequence translocase. Mol. Biol. Cell 19: 2642-2649.

Jameson BA and Wolf H 1988 The antigenic index: A novel algorithm for predicting antigenic determinants. Bioinformatics 4(1): 181-186.

Kampinga HH, Hageman J, Vos MJ, Kubota H, Tanguay RM, Bruford EA, Cheetham ME, Chen B and Hightower LE 2009 Guidelines for the nomenclature of the human heat shock proteins. Cell Stress Chaperones 14(1): 105-111.

Matuschewski K 2006 Getting infectious: Formation and maturation of plasmodium sporozoites in the Anopheles vector. Cellular Microbiology 8(10): 1547-1556.

Mendis K, Sina BJ, Marchesini P and Carter R 2001 The neglected burden of Plasmodium vivax malaria. Am. J. Trop. Med. Hyg. 64(1, 2) S: 97-106.

Molina-Cruz A and Barillas-Mury C 2014 The remarkable journey of adaptation of the Plasmodium falciparum malaria parasite to New World anopheline mosquitoes. Mem. 
Inst. Oswaldo Cruz 109(5): 662-667.

Muralidharan V, Oksman A, Pal P, Lindquist S and Goldberg DE 2012 Plasmodium falciparum heat shock protein 110 stabilizes the asparagine repeat-rich parasite proteome during malarial fevers. Nat. commun. 3: 1310 .

Murray CJL, Rosenfeld LC, Lim SS, Andrews $\mathrm{KG}$, Foreman KJ, Haring D, Fullman N, Naghavi M, Lozano R. and Lopez AD 2012 Global malaria mortality between 1980 and 2010: a systematic analysis. Lancet 379: 413-431.

Neupert W and Brunner M 2002 The protein import motor of mitochondria. Nat. Rev. Mol. Cell Biol. 3(8): 555-565.

Njunge JM, Ludewig MH, Boshoff A, Pesce ER and Blatch GL 2013 Hsp70s and J proteins of Plasmodium parasites infecting rodents and primates: Structure, function, clinical relevance, and drug targets. Curr. Pharm. Des. 19(3): 387-403.

Pavithra SR, Kumar R and Tatu U 2007 Systems analysis of chaperone networks in the malarial parasite Plasmodium falciparum. PLoS Comput. Biol. 3(9): e168.

Pérez-Morales D and Espinoza B 2015 The role of small heat shock proteins in parasites. Cell Stress Chaperones 20(5): 767-780.

Pesce ER and Blatch GL 2014 Plasmodial Hsp40 and Hsp70 chaperones: current and future perspectives. Parasitology 141(9): 1167-1176.

Powers ET, Morimoto RI, Dillin A, Kelly JW and Balch WE 2009 Biological and chemical approaches to diseases of proteostasis deficiency. Ann. Rev. Biochem. 78: 959-991.

Prudêncio M, Rodriguez A and Mota MM 2006 The silent path to thousands of merozoites: the Plasmodium liver stage. Nat. Rev. Microbiol. 4(11): 849-856.

Rathore S, Datta G, Kaur I, Malhotra P and Mohmmed A 2015 Disruption of cellular homeostasis induces organelle stress and triggers apoptosis like cell-death pathways in malaria parasite. Cell Death Dis. 6(7): e1803.

Rowe JA, Claessens A, Corrigan RA and Arman M 2009 Adhesion of Plasmodium falciparum-infected erythrocytes to human cells: molecular mechanisms and therapeutic implications. Expert Rev. Mol. Med. 11: e16.

Sherman IW 1998 A brief history of malaria and discovery of the parasite's life cycle. In: Sherman IW, editor. Malaria: Biology, pathogenesisand protection. Washington (D.C.): ASM Press.

Shonhai A Maier AG, Przyborski JM Blatch GL 2011 Intracellular protozoan parasites of humans: the role of molecular chaperones in development and pathogenesis. Protein Pept. Lett. 18: 143157.

Siregar JE, Kurisu G, Kobayashi T, Matsuzaki M, Sakamoto K, Mi-ichi F, Watanabe Y, Hirai M, Matsuoka H, Syafruddin D, Marzuki S and Kita K 2015 Direct evidence for the atovaquone action on the Plasmodium cytochrome bc1 complex. Parasitol. Int. 64(3): 295-300.

Snow RW 2015 Global malaria eradication and the importance of Plasmodium falciparum epidemiology in Africa. BMC Med. 13(1): 23.

Trager W and Jensen JB 1976 Human malaria parasites in continuous culture. Science 193(4254): 673-675.

Tilley L, Dixon MW and Kirk K 2011 The Plasmodium falciparum-infected red blood cell. Int. J. Biochem. Cell Biol. 43(6): 839842.

Trougakos IP 2013 The molecular chaperone apolipoprotein $\mathrm{J} /$ clusterin as a sensor of oxidative stress: Implications in therapeutic approaches-A mini-review. Gerontology 59(6): 514-523.

Vaughan AM, Kappe SHI, Ploss A and Mikolajczak SA 2012 Development of humanized mouse models to study human malaria parasite infection Ashley. Future Microbiol. 7(5): 657-665.

Vincensini L, Richert S, Blisnick T, Van Dorsselaer A, Leize-Wagner E, Rabilloud T 
Tanz. J. Sci. Vol 47(4) 2021

and Braun Breton C 2005 Proteomic analysis identifies novel proteins of the Maurer's clefts, a secretory compartment delivering Plasmodium falciparum proteins to the surface of its host cell. Molecular Cellular Proteomics 4(4): 582-593.

WHO 2012 World Malaria Report 2012, Accessed from: http://www.who.int/malaria/publications/w orld_malaria_report 2012/wmr2012_full_r eport.pdf

WHO 2015 World health statistics 2015. https://doi.org/ISBN9789241565257

Zofou D, Nyasa RB, Nsagha DS, Ntie-Kang F, Meriki HD, Assob JCN and Kuete V 2014 Control of malaria and other vector-borne protozoan diseases in the tropics: enduring challenges despite considerable progress and achievements. Infect. Dis. Poverty 3(1): $1-14$. 